\title{
Multiple realities?
}

\section{Marie Jahoda}

IN THE last quarter of the nineteenth century psychologists, impressed by the advances in the hard sciences, emulated their big brother and issued a unilateral declaration of independence from philosophy. Both, emulation and independence, were powerful motivators; they appeared to work reasonably well in mainstream psychology, even though doubts about the wisdom of either were raised from the beginning.

For a good part of the twentieth century emulation was based on the mechanistic physics of earlier periods. But physics had undergone revolutionary changes. Its new concepts - particularly Heisenberg's principle of uncertainty and the probabilistic nature of knowledge in many areas - gradually permeated the intellectual culture of psychologists, many of whom were all too aware of uncertainties in their methods, concepts and findings. References to Heisenberg increased in the psychological literature, used defensively however, rather than as a stepping stone to further advances, as was the case in physics.

The book under review, written by a research psychologist and a physicist, repeals the declaration of independence from philosophy in both disciplines and searches for a redefinition of their communalities and differences. It is organized in three parts, entitled "The Meanings of Reality", "The Search for Scientific Truth" and "Domains of the Social Sciences". Philosophers of science have dealt with such issues often as outsiders to the practice of science and have, as a rule, concentrated on the hard sciences; the reversed intrusion by practitioners of science into epistemology is largely limited to a specific field. The increase in books, of which this is one, that transcend both these limitations is a welcome sign of efforts to put the human sciences on a more solid base, even those aspects where matters count that cannot be counted; at least not yet.

In crude outline (omitting many interesting asides such as Heisenberg's appreciative comments on Goethe's theory of colours) the rather involved argument of the authors runs thus: the materialistic concept of reality has been shown to be limited by "the works of Heisenberg, Schrödinger, Einstein, Born, Freud, and Jung" (p.1) and needs reformulation. While common sense still regards as real only the external world that can be seen or touched, modern physics constructs as real

\section{Einstein's Space and Van Gogh's Sky: Physical Reality and Beyond. By Lawrence LeShan and Henry Margenau. Pp.268. ISBN 0-02-570460-5. (Macmillan, New York: 1982.) \$14.95. To be published in the United Kingdom in January 1983 by Harvester.}

events in the macro- and in the microcosm that are beyond the sensory competence of the human organism and can be understood only mathematically or through inference from observeable consequences. Newton's formulation of reality was not wrong but insufficient in view of later constructions that left room for his but transcended it. In particle physics and astrophysics visualization is no longer possible, but every physicist regards the regularities established in these areas as real. New constructs of reality have been invented and their correspondence to observation has been discovered.

Physics was for a time erroneously believed by some human scientists to have solved their problems. This is wrong. The aspect to be taken on board from modern physics is the idea that it is legitimate and, for certain areas, necessary to use alternate interpretations of reality, particularly when psychologists go beyond overt behaviour to study the domains of inner life. Here it is unavoidable to assume that purpose and intention are real, even though they can only be inferred (much like the electron), not seen or felt by an observer. This is a construction of psychic reality with which many psychologists have been familiar since the beginning of the century in a systematic way, but even St Augustine knew that psychological time had to be construed differently from measureable time, and accepted both as real.

Just as physics distinguishes between various domains (mechanics, quantum physics and so on) that are based on different constructions of reality, so the human sciences must identify their domains. Those suggested here in addition to molar behaviour are: art, parapsychology, ethics and consciousness. A curious assembly.

Ignoring this idiosyncratic choice of domains, what is one to make of all this? As a non-physicist I learn from this and other expositions of modern physics for the layman one important lesson: I have to take it on credit. As a non-philosopher I can only marvel at the amount of mental energy that has been expended over the centuries on pondering what reality really is: a dream, a unitary or multiple cosmos, a deliberate creation or an evolution by chance and necessity? These eternal ontological questions are metaphysics, not science. As a psychologist, I agree with the authors of this volume, that, whatever one's metaphysical stance "... we can never determine the 'true' shape and order of reality. We shall have to give up this dream" (p.24). All we can do qua scientists is to invent different ways of thinking about reality (including consciousness as much as the physical world) and check the extent to which concepts correspond to observation.

Two pages later, on p.26, the various constructs have changed into various essences, when the phrase is ". . . multiple, equally valid realities . . .", not constructs of reality. The very chapter title "Alternate Realities" implies the same deviation from p.24, and throughout the book there are other examples of confounding what there is with how we think about it. Surely we must assume that particles performed their indeterminate dance even before modern physics began to look at them as real?

It may seem pedantic to criticize the authors for what might be regarded only as elliptic phrasing, given that the basic position of an unending asymptotic approach to reality was stated clearly early on. But this is not the only case of loose talk about concepts crucial to the entire argument. Another is the concept of purpose, an indispensible concept in much of psychology where it is regarded as a state of consciousness in the here and now. On p.132, however, it is equated with "determination by the future"; on p.157 "future states influence present occurrences". This is magic. Once again, St Augustine and modern psychology know better. It is the present anticipation, not a future event which may or may not occur, that influences experience and action.

There are also some minor flaws: R.F. Bales is consistently misspelled; the index is not very helpful; some of the down-toearth examples are not convincing; and to usurp art and ethics for psychology seems to me misplaced imperialism. Altogether, an interesting intention has been disappointingly executed.

Marie Jahoda is Professor Emeritus of Social Psychology and Consultant to the Science Policy Research Unit at the University of Sussex. 